\title{
A Tale of Two Internet News Platforms-Real vs. Fake: An Elaboration Likelihood Model Perspective
}

\author{
Babajide Osatuyi \\ The University of Texas Rio Grande Valley \\ babajide.osatuyi@utrgv.edu
}

\author{
Jerald Hughes \\ The University of Texas Rio Grande Valley \\ j.hughes@utrgv.edu
}

\begin{abstract}
This paper presents findings from a field analysis of real vs. fake news propagated on the Internet. Elaboration Likelihood Model (ELM) was used as a theoretical framework to investigate information presentation mechanisms used by real and fake content generators to persuade readers. ELM theorizes two routes through which information can inform attitudinal changes: a central route of high cognitive effort, and a peripheral route of low cognitive effort. We hypothesize that fake news sites favor the peripheral route by providing less information overall, and by providing more negative affective cues. Data was gathered from Internet platforms that publish real news and fake news. Results indicate that the amount of information disseminated by fake news platforms is lower than that of reputable platforms. Content analysis reveals that fake news with business impact are typically more negative in their valence compared to real news. Implications of our findings for theory and practice are discussed.
\end{abstract}

Keywords: Fake news, elaboration likelihood model, information presentation, diffusion, persuasion

\section{Introduction}

Internet news platforms have become widely used sources of information about topics of interest and especially about organizations that people do business with directly or indirectly [1]. News platform designers take advantage of this wide audience by publishing what readers find to be attractive. However, online platforms may drive readership by propagating bad and sometimes fake news to keep readership up [2]. When the bad news is about businesses, the bad publicity may dissuade future potential customers from patronizing the business, leading to poor sales. Advancements in interactive technologies have exacerbated the generation and propagation of such fake content on the Internet [3]. Unlike the elaborate procedure associated with creating, validating and disseminating real news, fake news can be conjured in a manner of minutes and sent out to millions of customers instantaneously via the Internet.

Existing research on fake news presentation report the increasing success rates of fake news generators in persuading recipients to change their attitudinal responses in a desired direction [4]. It is commonplace in election campaigns for opposing candidates to successfully publicize fake news about their opponents to sway supporters their way [5]. Given the visible impacts of fake news, it is important to understand how fake news influences attitudes. Understanding the mechanisms responsible for successful presentation of fake news has some double-edged implications. On the one hand, armed with such understanding, organizations can quickly detect ill-targeted news towards the company and proactively respond by disseminating real information advising customers of the impending fake news. On the other hand, unscrupulous purveyors of online "news" with deep understanding of the persuasive mechanisms at work might utilize falsification of information in even more effective ways.

This study seeks to understand the mechanisms used to propagate fake news by using the Elaboration Likelihood Model (ELM) as a theoretical framework. This model has been used in IS research to investigate information presentation on the Internet across different contexts. ELM provides two routes (central and peripheral) through which a content generator can convince recipients to change their behavior in favor of a target persuasion. Data for this study largely support the routes proposed by ELM. Findings from this research are discussed with implications for practice and research.

The rest of the paper is presented as follows. In what follows (Section 2) is a review of the relevant literature on how information is presented to target audiences followed by a research model (in Section 3 ) that is developed based on ELM model. Hypotheses are developed in Section 4. The methods, result, and discussion sections are then presented in Sections 5, 6 and 7 respectively followed by the conclusion in Section 8 . 


\subsection{Literature Review}

Fake news presentation refers to the deliberate publication of news (or information) that is fabricated, unconfirmed or untrue leading to media bias $[6,7]$. The motivation for propagating fake news can range from monetary gain in the form of clickbait to the propagation of a political agenda to win the support of voters [7]. Regardless of the motivation, this study takes the position that it is important to investigate the presentation features utilized by fake content generators on the Internet due to their potential implication on business performance.

Most studies on understanding fake news presentation have been conducted in the communications field. Moreover, central to the findings is the facilitating role that information and communication technology plays in this propagation. Earlier research focused on the traditional communication channel such as TV shows $[8,9]$ while more recent studies examined the use of social media platforms $[5,10]$. The importance of information technology, particularly the Internet, in the presentation of fake news makes this topic of interest to IS researchers and serves as the motivation for this study.

Unfortunately, the literature demonstrates that fake news in online contexts is playing an important role in attitudinal development among some readers. Marchi [10] found that people show preference for fake [opinionated] news compared to objective curated news from the media. The researcher reported that the preference for opinionated news was due to the more authentic communication of the fake information compared to the communication of objective, real news. McBeth and Clemons [11] also found that fake news was considered by people to be more realistic than objective media news. These studies highlight recipients' attention to the appeal of the message rather than the credibility of the actual news content. A study of the impact of fake news presentation during the 2016 presidential election reported that the winning candidate had more fake news propagated during the three months leading to the election than the opponent [5]. More importantly, the study reported that a single fake news item propagated on social media had the same persuasive effect as 36 television campaign ads. Next, we review an information processing theory that can explain the mechanisms through which information can be disseminated with the intention to persuade recipients.

\section{Research Model}

\subsection{Theory: Elaboration Likelihood Model}

The elaboration likelihood model (ELM) of persuasion [12] was developed in the field of Psychology to understand how individuals develop or change their attitude about artifacts, people or events in their environment in order to inform their behavior about or toward those artifacts, people or events. ELM argues that it is imperative to consider two routes through which information may be conveyed to persuade its recipients, namely central and peripheral routes. The central route involves a systematic evaluation of the information received by a recipient. This route requires a considerable amount of cognitive effort on the part of the recipient to assess information received to develop a favorable or unfavorable response to the information. This means that attitude change in this route is dependent on the motivation and cognitive ability (or expertise) of the recipient to process the information to make the change decision. On the other hand, the peripheral route involves the use of associative memory to formulate their attitude change decision, with low cognitive effort needed. Attitude change is informed by the extent to which cues in the available information trigger an affective response that is associated with the persuasion context. Elaboration is said to be high when people use the central route to inform their attitude change whereas it is said to be low when people use the peripheral route to determine their attitude change.

ELM has been used in IS research in different contexts including health-IT adoption [13], enterprise resource planning adoption [14]. More recently, researchers have focused on extending it to explain privacy concerns $[15,16]$. While these studies focus on how individuals modify their attitudes to adopt technologies, research is scanty on the strategic persuasion in communication initiated by news media organizations [17].

\subsection{ELM model of Fake vs. Real News Presentation}

Our research model explores how Internet news platforms present information to their readers from an elaboration likelihood perspective. The review presented in the previous section suggests that Internet news platforms structure the delivery of their contents using two strategies (central and peripheral routes). Per ELM, inasmuch as a user is motivated and able to process the information presented on the platform, news platforms will deliver detailed information with high 
variance and less emphasis on affective cues such as those promoting a negative agenda.

Fake news platforms on the other hand use the peripheral route to pull the attention of the readers toward the presentation of the information rather than the merit of the information. Consequently, the strategy is to present information that is short in length and low in variance, since the focus is on catchy phrases, clichés and slogans, rather than on capturing the specific factual details which could support the veracity of the news being presented. Information presented using this strategy also tend to be more negative than real news because of media bias that presumes that negative news is more attractive to readers than real positive news.

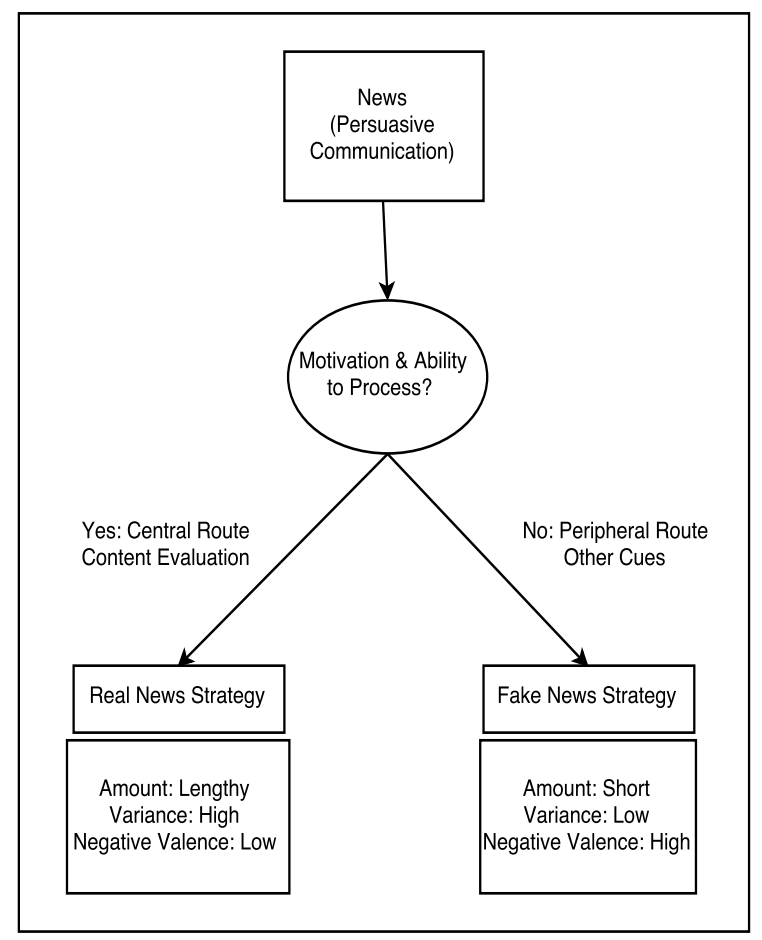

Figure 1. ELM Model of News Delivery

\section{Hypotheses}

\subsection{Amount of information communicated}

Because of the characteristics of the peripheral route described in ELM theory earlier, we predict that the amount of information communicated about a business on fake news platforms will be less than that of similar news on real news platforms. Fake news posts are targeted and concise because they are attention grabbers [1]. Real news on the other hand tend to be lengthy due to the detail provided. According to ELM, the central route is more elaborate, focused on systematic evaluation of information, so we expect that real news contain more information needed for the reader to process and build a case in support of the persuasion intended by the content provider.

Hypothesis 1. The amounts of information presented in posts on fake news platforms will be lower than those of real news

\subsection{Variance of information communicated}

The amount of information communicated about a business is limited in fake news compared to real news that provides detailed information. Fake news platforms only focus on providing enough false information to present a strong affective message for persuading readers. Space that might be used to establish the veracity of these false claims is literally not needed. This strategy follows the peripheral route in ELM that provides for the presentation of limited information, and focuses on specific, usually irrelevant, cues of the news platform. Real news on the other hand is presented systematically with facts and statements of validation to help the reader assess the credibility of the information. The amount of information needed here depends on the actual specifics of the story, since real news is dependent on real, verifiable facts. The presentation of elaborate information to convey the message and ascertain its credibility leads real news platforms to share a wide variety of information about the topic, methodically developing the case for the factual reliability and therefore real news value of the post. Conversely, fake news, if following the peripheral route, can neglect such details, and thus be relatively unresponsive to the differences in the amounts of relevant information which are important for news posts based on actual facts. Fake posts are rather constrained in length by the imperative to motivate the reader to react quickly, without the need for reading large amounts of detail. Therefore:

Hypothesis 2. The variances of amounts of information presented in posts on fake news platforms will be lower than those of real news

\subsection{Valence of information communicated}

News often tend toward negativity in tone [18]. Negative tone is attractive to readers and makes catchy headlines which translate to a wider audience and consequently increase revenue for the news provider [2]. Fake news platforms, in their attempt to quickly appeal to a wide audience, emphasize a particularly negative and sensational tone. 
Fake news is generally attention-grabbing and requires less cognitive effort from the recipient to make sense of the information. The goal is to draw the attention of readers to immediately react to a collection of affective words or phrases rather than read a paragraph-long description. According to ELM, fake news will need to be crafted in such a way as to encourage its recipients to take the peripheral route to make sense of the information quickly. ELM posits that when a recipient is not motivated and/or does not have the cognitive capacity to make sense of complex information, they resort to using the peripheral route to facilitate their decision making. For example, marketing research is replete with strategies to reel-in customers by showing a big sale sign to attract them and in the form of popups, ads and emails.

Although findings from prior research suggest that news media in general tend to broadcast more negative than positive news, the majority of their research focus was on politics. In the context of news that impact businesses, the economic and reputation implications of news platforms depend on the information that will generate a large audience [2]. The dissemination of negative news about businesses has been shown to be widely adopted for a longer time than positive news [3]. Due to their reliance on the peripheral route, we expect that when Internet fake news platforms communicate news that have business impacts, they will focus on using a higher number of negatively toned words, as compared to the terms found in real news posts. We will consider this prediction from two related perspectives: scores against all the words in each post, and scores against the vocabularies used in each post. We therefore present two similar but distinct hypotheses.

Hypothesis 3. The negative valence of information presented in the full texts of posts on fake news platforms will be higher than that presented on real news platforms

As a related indicator of negative valence, we theorize that not only will fake news sites have higher negative valence scores in the full texts of the posts; they will also depend systematically on vocabularies which have higher negative valence than those of real news posts. That is, the higher negative valence of fake news posts will continue to be observed, even when repetitions of specific negative words are neglected.

Hypothesis 4. The negative valence of the vocabularies used in posts on fake news platforms will be higher than those used on real news platforms

\section{Method}

To examine how news (real and fake) with bad business impacts are presented on the Internet, we analyzed news posted by real and fake news platforms about businesses. In order to provide analyses with the broadest possible implications for where fake news is transmitted, we have focused solely on the inherent textual characteristics of the fake news itself. These characteristics are thus independent of whether the text appears in a commercial website, a blog, a forum, a comment, or social media platforms. Wherever the actual texts are reproduced, the measures we employ will apply. Particularly because the nature of the threats posed by fake news depend on their viral spread across diverse platforms on the Internet, we consider increased understanding of the texts themselves as particularly important.

In the course of first examining fake news, we encountered an enormous number of political posts, as well as items focused on the paranormal, UFO's, the disgusting, and the simply weird or bizarre; all of these may be presumed to be valuable for fake news sites because of their immediately sensational nature. For our purposes in producing research of interest to businesses and organizations with reputations and brands to protect, we decided to focus strictly on posts which presented bad news about organizations identified explicitly by name in the post. These pose the most direct threat to businesses, and provide a well-defined narrow scope around which to focus our study. Our data is thus free of potential confounding features which might arise due to differences in how the different fake news topics listed above might be treated in their manner of presentation.

\subsection{Data collection and procedure}

In order to develop a sample which could best address the aspects of comparison in the hypotheses above, we reduced potentially confounding influences by carrying out a sequence of steps to identify the best possible sources for comparison. Data for this study was thus collected using following the steps.

Step 1: As a preliminary scan, we identified news posts confirmed as fake news, about Fortune 500 businesses, which are bad for the business.

Step 2: We submitted the identified business names from Forbes 500 and other sources to the "Fact Check" function on snopes.com, for 'False' ratings, yielding 23 sources of bad news confirmed to be fake. Confirmations that the 'news' was false were typically observed not only on snopes.com, but across multiple 
fact-checking sites. This step thus established that the posts in question were actually false.

Step 3: We examined the fake sources from steps 1 and 2 for additional bad business news items. Most sites published fake news of multiple types, not just business, so not all fake sources actually provided as many as 10 stories with bad news about businesses identified by name. Inclusion of only those sites with at least 10 bad news stories about businesses led the identification of 8 confirmed 'Fake' sources that contained multiple instances of the precise type of information needed for our analysis.

Step 4: We then collected 10 news posts from each of the fake sources. These posts were likewise restricted to news which reflected badly on the organizations at the center of the story.

Step 5: We then identified highly reliable news sources largely based on Forbes report of 10 reliable sources used (e.g., Associated Press, CNN, New York Times, BBC, etc.). 8 'Real' sources were identified to match up with the sources of fake news. Sources on the Forbes list which were magazines such as Economist and New Yorker were not used, because their story formats, derived from their print versions, tend toward much longer treatments we considered not comparable for the purposes of the desired sample.

Step 6: For each of the real sources, we collected 10 samples of real news which corresponded as closely as possible to the fake stories used. Example: fake rat meat stories were collected for fake sites; real sites were scanned for stories about rat meat-real bad news for the organizations involved.

The fake and real data sets thus contain 80 samples each. These two sets are matched on: 1) business or organization news (not politics, paranormal, weird/gross, celebrities), 2) bad news, 3) same topic or one closely related. Example: One fake news story is about bananas with HIV microbes; the real site story is about bananas with other blights, or contaminated fruit, and 4) from sites confirmed as Fake, or confirmed as Real.

\subsection{Measurement items}

A summary of the measurement items extracted from the data collection procedure is presented in Table 1. Amounts of information were determined by calculating the Shannon information entropy in bits for each post, using individual words as the unit of counts employed in the entropy calculations. In order to remove the 'noise' introduced by function words (a, and, of, etc), we calculated this measure for Non-Function Words only, thus improving the focus on actual semantic information present. For all analyses below, the calculated results are performed on the texts of each posts with the function words (non-semantic words) removed.

Table 1. Summary of Measurement Items.

\begin{tabular}{|l|l|}
\hline $\begin{array}{l}\text { Variable } \\
\text { categories }\end{array}$ & \multicolumn{1}{|c|}{ Measurement } \\
\hline $\begin{array}{l}\text { Amounts of } \\
\text { information }\end{array}$ & $\begin{array}{l}\text { Shannon information entropy } \\
\text { expressed in bits for each post, using } \\
\text { word counts as unit of Shannon } \\
\text { calculation }\end{array}$ \\
\hline $\begin{array}{l}\text { Variance in } \\
\text { amounts of } \\
\text { information } \\
\text { by source }\end{array}$ & $\begin{array}{l}\text { Variance in Shannon information } \\
\text { entropy bits for each post; variance } \\
\text { calculated separately for each of the } \\
16 \text { news sources }\end{array}$ \\
\hline $\begin{array}{l}\text { Negative } \\
\text { valences of } \\
\text { full posts }\end{array}$ & $\begin{array}{l}\text { Scoring of each post's full text } \\
\text { based on counts of terms of negative } \\
\text { polarity in Opinion Lexicon of Hu } \\
\text { and Liu [19] }\end{array}$ \\
\hline $\begin{array}{l}\text { Negative } \\
\text { valences of } \\
\text { vocabularies } \\
\text { of posts }\end{array}$ & $\begin{array}{l}\text { Scoring of each post's vocabulary } \\
\text { based on counts of terms of negative } \\
\text { polarity in Opinion Lexicon of Hu } \\
\text { and Liu [19] }\end{array}$ \\
\hline
\end{tabular}

\subsection{Analysis}

Because the posts for the fake versus real news are not individually paired, they were analyzed as independent samples, divided between fake news sources and real news sources. The data meets the normality assumption requirements for the analyses required to test our hypotheses. T-tests were conducted to test our hypotheses, since they all posit relationships in which one variable is greater than the other. Onetailed t-tests were used because the differences in the observed means are all hypothesized in a specific direction. In addition to the significance tests, we also report effect sizes [20]. According to [20], the effect size is small if Cohen's D is at least 0.20 , medium if it is at least 0.50 and large if is at least 0.80 . Procedures were as follows.

For hypothesis 1, the amount of Shannon information entropy bits was calculated for each individual post. Since the units of interest are semantic, word counts (not individual letter character counts) were used as the basis for entropy calculations.

For hypothesis 2, we theorize a difference based on the notion that the precise details of an incident are important for real news producers (ELM central route), but relatively incidental to fake news sources (ELM peripheral route). Thus, a given real news source should exhibit greater variance in its amounts of information presented from story to story, because the details required to present real news depend on the specifics of 
each story. Conversely, fake news stories, depending on ELM peripheral cues, are expected to present less variance in information amounts, since precise content details are not of concern. The unit of variance is the particular source (e.g., Associated Press). To capture variances separately for each source, we measured the Shannon information entropy bits for each individual post, then calculated the variances of that measure among the 10 posts captured for each source. This yielded 8 variances of fake sources, and 8 variances of real sources. These two groups were then subjected to ttest for comparison of the means of the variances of the Shannon entropy bits.

For hypothesis 3 , we theorized that fake news posts will be more negative than real news. To test this hypothesis we obtained a list of 4783 terms of negative polarity provided for the purpose of determining negative valence by Minqing $\mathrm{Hu}$ and Bing Liu of the University of Illinois at Chicago [19]. For each post, we counted first the number of total non-function words, then the number of terms from the negative polarity list present in the same post; this constituted a raw score. Because posts varied considerably in length, we removed the confounding effect of differing lengths by dividing each raw score by the number of total words present; this yields a scaled negative valence score for each post which represents the density of negative terms in each post. The two groups, fake and real, were then subjected to t-test for difference of means of these scores. For hypothesis 4, we provided a related but distinct analysis, in this case scoring the vocabularies of each post, instead of the full texts. The procedures were otherwise the same.

\section{Results}

The results of our analyses and significance tests are summarized in Table 2. Hypothesis 1 tested the difference in amounts of information, measured as bits, between Fake and Real news posts. For this measure, Levene's test for homogeneity of variance indicated that the hypothesis that variances of the two groups were equal could not be rejected (on means: 0.576), so we report results assuming equal variances. Our results indicate that the average amount of information in the fake news posts is less than the average amount of information on real news posts $(p<0.001)$. The effect size (Cohen's D) of this difference test is 1.329 , well above the threshold value for large effect size (0.80).

Hypothesis 2 tested the difference in the variances of information in posts between fake and real news platforms. Levene's test performed on the two groups of sources indicated that the hypothesis that variances of the two groups were equal should be rejected (on means: 0.0292), so here we report the results assuming unequal variances. The results indicate that variance in the news from real news sources is larger than that of fake news sources. The effect size is very large (Cohen's $D=1.65$ ) This reflects the notion that real news sites are concerned with communicating the meaningful and reliable content of each specific story, which may vary considerably, whereas Fake posts have a more uniform size, because these platforms do not motivate their readers through factual content details.

Hypothesis 3 tested the difference in negative valence between fake and real news post texts. Levene's test indicated that variances were not homogeneous (on means: 0.0047), so here we report the results assuming unequal variances. The results support our hypothesis that the average scaled negative scores of fake news platforms is greater than that of real news. The effect size of this difference is on the small-tomedium side (Cohen's $\mathrm{d}=0.448$ ), but still significant.

Hypothesis 4 tested the difference in negative valence between fake and real news post vocabularies.

Table 2. Results across real and fake news platforms.

\begin{tabular}{|c|c|c|c|c|c|c|c|c|}
\hline Hypothesis & Measures & Type & Count & Mean & t-stat & t-crit & p-value & Cohen's D \\
\hline \multirow{2}{*}{ H1 } & \multirow{2}{*}{$\begin{array}{l}\text { Information } \\
\text { Amounts }\end{array}$} & Fake & 80 & 6.91 & $8.41 * * *$ & 1.655 & $<0.001$ & 1.329 \\
\hline & & Real & 80 & 7.56 & & & & \\
\hline \multirow{2}{*}{ H2 } & \multirow{2}{*}{$\begin{array}{ll}\text { News } & \text { source } \\
\text { variances } & \\
\end{array}$} & Fake & 8 & 0.094 & $3.25 * *$ & 1.895 & 0.0029 & 1.625 \\
\hline & & Real & 8 & 0.246 & & & & \\
\hline \multirow{2}{*}{ H3 } & \multirow{2}{*}{$\begin{array}{l}\text { Negative Valences of } \\
\text { full texts }\end{array}$} & Fake & 80 & 0.044 & $2.83 * *$ & 1.655 & 0.0026 & 0.448 \\
\hline & & Real & 80 & 0.036 & & & & \\
\hline \multirow{2}{*}{ H4 } & \multirow{2}{*}{$\begin{array}{l}\text { Negative Valences of } \\
\text { Vocabularies }\end{array}$} & Fake & 80 & 0.060 & $1.72 *$ & 1.655 & 0.0432 & 0.273 \\
\hline & & Real & 80 & 0.054 & & & & \\
\hline
\end{tabular}

$* * *$ : Significant at $\alpha=.001 ; * *$ : Significant at $\alpha=.01 ; *$ : Significant at $\alpha=.05$ 
The results support our hypothesis that fake news posts' vocabularies are more negative than those of real news posts, this time with a small effect reflected in the Cohen's D.

Table 3 shows that all the hypothesized relationships in the study were supported. Hypotheses 1,2 and 3 were highly significant, while hypothesis 4 was significant but with higher $p$-value than the rest. Hypotheses 1 and 2 exhibited very large effects, 3 had a small-medium effect, and 4's effect was small.

Table 3. Summary of Hypotheses Tests.

\begin{tabular}{|l|c|}
\hline Hypothesis & Supported? \\
\hline $\begin{array}{l}\text { Hypothesis 1. The amounts of } \\
\text { information presented in posts on } \\
\text { fake news platforms will be lower } \\
\text { than those of real news }\end{array}$ & Yes \\
\hline $\begin{array}{l}\text { Hypothesis 2. The variances of } \\
\text { amounts of information presented in } \\
\text { posts on fake news platforms will be } \\
\text { lower than those of real news }\end{array}$ & Yes \\
\hline $\begin{array}{l}\text { Hypothesis 3. The negative valence } \\
\text { of information presented in the full } \\
\text { texts of posts on fake news } \\
\text { platforms will be higher than those } \\
\text { presented on real news platforms }\end{array}$ & Yes \\
\hline $\begin{array}{l}\text { Hypothesis 4. The negative valence } \\
\text { of the vocabularies used in posts on } \\
\text { fake news platforms will be higher } \\
\text { than those used on real news } \\
\text { platforms }\end{array}$ & Yes \\
\hline
\end{tabular}

\section{Discussion}

This study examined differences in the characteristics of news that have bad business impacts on the Internet posted by fake and real news platforms ${ }^{1}$. All the hypotheses proposed were supported, leading to the conclusion that in general, fake news platforms present news that has bad business impacts in ways which are systematically different from those of real news sources.

The results are consistent in supporting the idea that fake news posts are systematically more aligned towards low elaboration, in which readers exert lower cognitive effort to assess information, reacting instead to affective cues. The strongest effects measured were those reflecting the lower amounts of information

\footnotetext{
1 The determination of the negative business impact of news information posted on the Internet was computed by
}

provided by fake news. The reduced amounts of information in fake posts facilitate the quicker processing typical of the peripheral route identified in ELM theory.

Furthermore, the affective cues typical of the peripheral route to attitudinal change in ELM theory appear to be well-represented in fake news. Not only are negative valence scores higher, reflecting higher density of negative terms, but fake sites also present more negative vocabularies. These cues provide fake news readers with the means by which to process the posts quickly, without the cognitive effort required of the central route. And we saw further indications in the bias of fake news posts toward affective cues.

Fake news platforms on the Internet typically aim to posts news about businesses in ways that are misleading but highly attention-getting, and easy for readers to understand. Consistent with the affective peripheral route characteristics posited in ELM, fake news platforms focus on highlighting the most negative implications. In order to explore this finding further, we extracted the top 20 most-used negative terms appearing in fake news, versus the top 20 negative terms of real news. The resulting lists provide a rather stark comparison. We have bolded the most prominent examples:

Table 4. Most-used negative terms

\begin{tabular}{|l|l|}
\hline $\begin{array}{l}\text { Top 20 Neg. Terms- } \\
\text { Fake }\end{array}$ & $\begin{array}{c}\text { Top 20 Neg. } \\
\text { Terms-Real }\end{array}$ \\
\hline hate & limit \\
allegations & breach \\
sued & broken \\
hell & stolen \\
horrifying & unlikely \\
refusing & worried \\
pain & trouble \\
sickening & complaints \\
rotten & losses \\
malicious & failure \\
slander & violation \\
urgent & criticism \\
aches & criticized \\
chaos & slow \\
shame & expensive \\
dumped & cheap \\
deceitful & apocalypse \\
dies & unknown \\
infamous & outcry \\
worries & complained \\
\hline
\end{tabular}

platforms such as snopes.com that use Fact-check procedures to estimate the negative implication of news information about businesses. 
As demonstrated in the Table 4, fake news platforms go the extra mile to use sensational, highly negative terms in their news posts about businesses. Real news platforms on the other hand are notably lacking in such sensational terms. This is consistent with our statistical finding in hypothesis 4 that fake news sites have a more negative vocabulary, considered this time in the qualitative sense of examples from the top 20's such as "hate", "hell" in the fake list, versus the much milder examples "limit", "breach" from the real list. Although news posts generally do tend toward negative in their tone, we provide empirical support for a higher proportion of negative words used by fake news platforms compared to real news platforms, even when considering only bad business news on both.

\subsection{Implications}

This research contributes to ELM and enterprise reputation management research. From a theoretical perspective, we extend ELM to explain the strategies used by real and fake news platforms to present news that has negative business impacts. We further contribute and demonstrate the use of methods of automated text analysis which, as pure content analyses, are independent of the particular Internet platforms used to present fake news. The theory and methods used here can be applied to fake news texts wherever they appear.

The ease and speed of creating and propagating fake news is alarming and presents a threat to the corporate image and competitiveness of an organization if it is not detected and corrected in a timely fashion. From a practical perspective, managers can use our findings to develop strategies to proactively detect fake news about their organizations and correct misinformation accordingly.

\subsection{Limitation and future research}

This research has some limitations that may influence the interpretation of our results. First, data for this study is based on a deliberately narrowly focused sample of news with bad business impacts from 8 specific fake news platforms. This was done in order to reduce potential confounding factors such as variances due to possible inconsistencies in the ways that the writers of such posts might address different topics, non-business news, good versus neutral versus bad news, etc. Our decision was to strengthen the validity of the conclusions drawn as much as possible by ensuring that the samples for comparisons chosen shared as many characteristics as possible. A future direction could include the collection of additional data on additional companies, from additional news sources. This would allow further segmentation, such as categories based on their position in the Fortune 500 list, or on the industries represented, the nations, etc. The next set of analyses could then for example explore how fake and real news platforms present news posts about companies at the top of the list compared with those in the middle and the bottom of the list. Second, this study does not necessarily generalize to the most numerous posts we observed, which were heavily political. Although we suspect that the language of fake political news is at least as emotive as that of fake business news, that point remains to be developed via further studies of negative valence.

\section{Conclusion}

This study sought to examine how real and fake Internet news platforms disseminate news that have business impacts. Using the ELM as a theoretical framework, we identified dissemination strategies as central and peripheral routes that Internet news platforms employ to persuade their readers. Data based on news posts about a sample of well-known organizations was used to test our hypotheses on the differences between the information presentation strategies used by Internet news platforms. Our results indicate that fake news platforms on average provide less information than real news platforms on the Internet. Findings from this study present practical and theoretical implications that are helpful for assessing fake vs. real news about businesses.

\section{References}

[1] A. Ju, S.H. Jeong, and H.I. Chyi, Will social media save newspapers? Examining the effectiveness of Facebook and Twitter as news platforms. Journalism Practice, 2014. 8(1): p. 1-17.

[2] S.N. Soroka, Good news and bad news: Asymmetric responses to economic information. Journal of Politics, 2006. 68(2): p. 372-385.

[3] J. Hornik, et al., Information dissemination via electronic word-of-mouth: Good news travels fast, bad news travels faster! Computers in Human Behavior, 2015. 45: p. 273-280.

[4] M. Jia, H. Ruan, and Z. Zhang, How rumors fly. Journal of Business Research, 2017. 72(1): p. 33-45.

[5] H. Allcott and M. Gentzkow, Social media and fake news in the 2016 election. 2017, National Bureau of Economic Research. 
[6] S. DellaVigna and E. Kaplan, The Fox News effect: Media bias and voting. The Quarterly Journal of Economics, 2007. 122(3): p. 1187-1234.

[7] T. Groseclose and J. Milyo, A measure of media bias. The Quarterly Journal of Economics, 2005. 120(4): p. 11911237.

[8] S.L. Borden and C. Tew, The role of journalist and the performance of journalism: Ethical lessons from "fake" news (seriously). Journal of Mass Media Ethics, 2007. 22(4): p. $300-314$

[9] A. Day and E. Thompson, Live from New York, it's the fake news! Saturday Night Live and the (non) politics of parody. Popular Communication, 2012. 10(1-2): p. 170-182.

[10] R. Marchi, With Facebook, blogs, and fake news, teens reject journalistic "objectivity". Journal of Communication Inquiry, 2012. 36(3): p. 246-262.

[11] M.K. McBeth and R.S. Clemons, Is the Fake News the Real News? The Significance of Stewart and Colbert on Democratic Discourse, Politics, and Policy, in The StewartColbert Effect: Essays on the Real Impact of Fake News (2011), A. Amarasingam, Editor. 2011, McFarland and Company.

[12] R.E. Petty and J.T. Cacioppo, The elaboration likelihood model of persuasion. Advances in experimental social psychology, 1986. 19: p. 123-205.

[13] C.M. Angst and R. Agarwal, Adoption of electronic health records in the presence of privacy concerns: The elaboration likelihood model and individual persuasion. MIS quarterly, 2009. 33(2): p. 339-370.

[14] C.-Y. Li, Persuasive messages on information system acceptance: A theoretical extension of elaboration likelihood model and social influence theory. Computers in Human Behavior, 2013. 29(1): p. 264-275.

[15] J. Gu, et al., Privacy concerns for mobile app download: An elaboration likelihood model perspective. Decision Support Systems, 2017. 94: p. 19-28.

[16] T. Zhou, Understanding location-based services users' privacy concern: an elaboration likelihood model perspective. Internet Research, 2017. 27(3): p. doi: 10.1108/IntR-04-2016-0088.

[17] K.Y. Tam and S.Y. Ho, Web personalization as a persuasion strategy: An elaboration likelihood model perspective. Information systems research, 2005. 16(3): $\mathrm{p}$. 271-291.

[18] S. Aday, Chasing the bad news: An analysis of 2005 Iraq and Afghanistan war coverage on NBC and Fox News Channel. Journal of Communication, 2010. 60(1): p. 144164.
[19] B. Liu and M. Hu. Opinion Mining, Sentiment Analysis, and Opinion Spam Detection. 2004 [cited 2017 May 10]; Available from: https://www.cs.uic.edu/ liub/FBS/sentiment-analysis.html.

[20] J. Cohen, Statistical power analysis for the behavioral sciences. Erlbaum. Hillsdale, NJ, 1988. 